\title{
Les effets des changements climatiques sur la santé et le bien-être dans les régions rurales et éloignées au Canada : synthèse documentaire
}

\author{
Amy Kipp, M.A. (1); Ashlee Cunsolo, Ph. D. (2); Kelly Vodden, Ph. D. (3); Nia King, B. Sc. (4); Sean Manners, B.A. (3); \\ Sherilee L. Harper, Ph. D. (1)
}

Diffuser cet article sur Twitter

\begin{abstract}
Résumé
Cet article présente une synthèse de la version préliminaire du chapitre à venir "Collectivités rurales et éloignées » de l'Évaluation nationale des changements climatiques du gouvernement du Canada, et il répertorie les principales préoccupations en matière de santé exposées dans la littérature sur les changements climatiques à propos des régions rurales et éloignées, ainsi que les stratégies d'adaptation actuelles et celles à mettre en place. Cet aperçu, fondé sur un processus de recherche systématique, souligne l'importance de tenir compte des composantes socioculturelles, économiques et géographiques spécifiques de ces régions ainsi que de l'expertise dont disposent déjà leurs habitants et leurs collectivités si l'on veut contribuer à la santé et au bien-être des populations qui subissent les effets nocifs des changements climatiques.
\end{abstract}

Mots-clés : changements climatiques, santé, bien-être, régions rurales, régions éloignées, adaptation, Canada

\section{Introduction}

Dans les régions du Canada rurales et éloignées (encadré 1), le rapport intime qu'entretiennent les individus et les collectivités avec leur environnement social, culturel et physique a souvent une influence importante sur la santé et le bien-être des populations ${ }^{1-5}$. Les habitants de ces collectivités dépendent souvent étroitement de leur environnement pour leur subsistance et leurs pratiques culturelles, ce qui a une incidence sur les déterminants sociaux de leur santé et de leur bien-être ${ }^{1-3,5,6}$. Les changements climatiques ont de ce fait des effets à la fois directs et indirects sur la santé et le bien-être des individus et des collectivités ${ }^{1,2,5,7,8}$.
Cet article présente une synthèse des données sur la santé contenues dans le chapitre «Collectivités rurales et éloignées » de la version préliminaire de l'Évaluation nationale des changements climatiques que publiera le gouvernement du Canada et qui s'intitulera Le Canada dans un climat en changement : renforcer nos connaissances pour mieux agir ${ }^{9}$. Au sein de cette évaluation, le chapitre sur les collectivités rurales et éloignées figure au côté d'autres chapitres consacrés à des enjeux nationaux - en particulier " Notre capital naturel », « Les dimensions internationales » et "Résilience de notre population et de notre société »- et de chapitres consacrés aux régions, soit " Nord du Canada », " ColombieBritannique », " Provinces des Prairies », « Ontario », " Québec » et « Provinces de
Points saillants

- Les changements climatiques ont des effets nocifs sur la santé et le bien-être des habitants et des collectivités des régions rurales et éloignées du Canada.

- Les principales préoccupations en matière de santé mises en évidence dans le chapitre "Collectivités rurales et éloignées » de l'Évaluation nationale des changements climatiques incluent l'exacerbation de problèmes liés à la sécurité des aliments et de l'eau, aux maladies chroniques, aux maladies infectieuses, aux blessures et décès accidentels et à la santé mentale.

- Bien que diverses caractéristiques spécifiques des collectivités rurales et éloignées soient à l'origine de leur vulnérabilité aux changements climatiques, ces régions disposent aussi de nombreux atouts soutenant leur résilience vis-à-vis de ces changements.

- Se centrer sur l'adaptation aux changements climatiques et prendre la mesure des avantages connexes résultant de l'atténuation de ces changements offre des opportunités au secteur de la santé.

\section{Rattachement des auteurs :}

1. École de santé publique, Université de l'Alberta, Edmonton (Alberta), Canada

2. Institut Labrador, Université Memorial, Happy Valley-Goose Bay (Labrador), Canada

3. Environmental Policy Institute, Campus Grenfell, Memorial University, Corner Brook (Terre-Neuve-et-Labrador), Canada

4. Faculté de médecine, Université Queen's, Kingston (Ontario), Canada

Correspondance : Amy Kipp, Université de l'Alberta, 11405, 87 avenue N.-O., Edmonton (Alb.) T6G 1C9; courriel : kipp@ualberta.ca

Ashlee Cunsolo, Institut Labrador de I'Université Memorial, B.P. 490, Station B, Happy Valley-Goose Bay (T.-N.-L.) A0P 1E0; tél. : 709-896-4702; courriel : ashlee.cunsolo@mun.ca

Sherilee L. Harper, École de santé publique, Université de l'Alberta, 11405, 87e avenue N.-O., Edmonton (Alb.) T6G 1C9; tél. : 780-492-7766; courriel : sherilee.harper@ualberta.ca 
ENCADRÉ 1

Définition des régions rurales et éloignées

Nous nous sommes appuyés sur la définition de l'ASPC ${ }^{31}$ pour définir les régions rurales et éloignées : ce sont des régions comptant moins de 10000 habitants. En outre, une collectivité rurale verra moins de $50 \%$ de sa population se déplacer vers une zone urbaine pour le travail, alors qu'une collectivité éloignée soit ne comptera aucun habitant se déplaçant vers une zone urbaine pour le travail, soit relèvera de l'un des territoires du Canada.

l'Atlantique »*. L'Évaluation va constituer une importante ressource pour les collectivités, les décideurs et le milieu universitaire en matière de décisions et de mesures relatives à l'adaptation aux changements climatiques. Elle offre une exploration de l'évolution du climat au Canada, des effets de cette évolution sur les Canadiens et des stratégies d'adaptation à employer pour réduire les risques climatiques ${ }^{9}$. Le chapitre sur les collectivités rurales et éloignées et, par extension, cet article reposent sur une analyse de la littérature grise et de publications évaluées par des pairs ${ }^{\dagger}$, sur une coopération active avec les chercheurs, les gouvernements, les collectivités, divers organismes et les chefs et gardiens du savoir autochtones et sur une collaboration avec les équipes rédigeant les autres chapitres de l'Évaluation. L'analyse bibliographique a permis de caractériser la nature, l'ampleur et la portée de la documentation publiée depuis 2013 (date de la dernière évaluation) au sujet des impacts des changements climatiques et de l'adaptation à ces derniers dans les collectivités canadiennes rurales et éloignées. Les volets quantitatif et qualitatif de cette analyse bibliographique ainsi que les données obtenues grâce aux diverses collaborations ont permis de définir les principaux enjeux de santé associés aux changements climatiques ainsi que les stratégies d'adaptation actuelles et celles à adopter pour ces régions. Notre article offre un aperçu de ces enjeux.

\section{Les changements climatiques, la santé et le bien-être dans les régions rurales et éloignées}

De nombreux changements climatiques et leurs répercussions environnementales ont un effet négatif sur la santé et le bien-être des habitants et des collectivités des régions rurales et éloignées, en particulier l'augmentation des phénomènes météo-

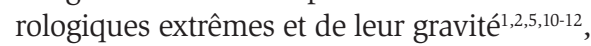
la modification des glaces maritimes, de la végétation, des espèces de poisson, de la faune en général et de l'eau ${ }^{1,2,5,12,13}$ ainsi que des incertitudes quant au climat et à l'environnement $t^{1,2,6,14}$.

Les principaux effets nocifs pour la santé associés à ces changements sont une incidence accrue de la malnutrition, de l'obésité et du diabète ${ }^{5,15,16}$, des maladies à transmission vectorielle ou d'origine hydrique ou alimentaire ${ }^{5,12,16,17}$, des maladies cardiovasculaires $^{15,16}$, des troubles respiratoires ${ }^{18}$, des blessures et des décès ${ }^{12-14}$ et des problèmes de santé mentale ${ }^{3,6,18-20}$. Certaines des caractéristiques des régions rurales et éloignées, dont l'isolement géographique et le caractère restreint des infrastructures de transport, une dépendance aux ressources naturelles et un manque de ressources au sein des infrastructures sociales et matérielles, sont susceptibles de contribuer à accroître la vulnérabilité à ces risques sanitaires ${ }^{2,5}$. Cette vulnérabilité aux changements climatiques relève aussi de la conjugaison de facteurs sociaux, culturels et politiques propres à ces régions et des caractéristiques et situations personnelles des populations ${ }^{2,5,21,22}$. D'après la synthèse émanant de notre analyse bibliographique, les principaux enjeux en matière de santé dans les régions rurales et éloignées sont : 1) des difficultés d'accès à une alimentation et à une eau de qualité, 2) une exacerbation des maladies chroniques et une incidence accrue des maladies infectieuses, 3) un risque accru de blessures et de décès accidentels, 4) des problèmes accrus en matière de santé mentale et de bien-être. En outre, le fait d'être ou non Autochtone, l'âge, le sexe et le statut socioéconomique seraient, d'après la littérature examinée, d'importants facteurs influençant la vulnérabilité aux changements climatiques dans les régions rurales et éloignées.

\section{Dégradation de la facilité d'accès à l'eau et à des aliments nourrissants et traditionnels}

De nombreuses régions rurales et éloignées connaissent une dégradation de l'accès aux circuits alimentaires et aux réseaux d'alimentation en eau, ainsi qu'une diminution de la qualité de ces circuits et réseaux, du fait de changements environnementaux comme la hausse des températures ${ }^{7,16,20,23}$, la transformation des régimes de précipitations et la multiplication d'événements météorologiques extrêmes ${ }^{7,18,23}$. Par exemple, dans de nombreuses communautés autochtones et inuites du Nord situées en région éloignée, les perturbations des glaces maritimes, de la faune et de la flore entraînées par les changements climatiques nuit à la possibilité de s'adonner à la chasse, à la pêche et à la cueillette, ce qui réduit la consommation d'aliments locaux sains et traditionnels et accroît la dépendance envers une alimentation industrielle $7,16,18,19,23$. De plus, la sécurité de l'eau, incluant l'accès, la disponibilité et la qualité, est compromise dans les régions rurales et éloignées, car la hausse des températures et la multiplication des événements météorologiques extrêmes peuvent à tout moment causer une surcharge des stations de traitement de l'eau, interrompant l'alimentation en eau potable $e^{7,23}$. Dans l'ensemble du Nord du Canada, où de nombreuses collectivités dépendent des sources d'eau de surface, les modifications des niveaux d'eau, du ruissellement, du régime d'écoulement et de l'accumulation des sédiments peuvent avoir des conséquences dramatiques sur l'accès à une eau potable de qualitée ${ }^{23,24}$. On sait que l'insécurité alimentaire et la pénurie d'eau sont corrélées à des effets nocifs sur la santé, en particulier de la malnutrition, de l'obésité, du diabète, des maladies cardiovasculaires, des maladies gastro-intestinales aiguës et des problèmes de santé mentale $\mathrm{m}^{7,12,16,25}$

\section{Exacerbation des maladies chroniques et incidence accrue des maladies infectieuses}

La transformation des régimes de précipitations, la hausse des températures et la multiplication de phénomènes météorologiques

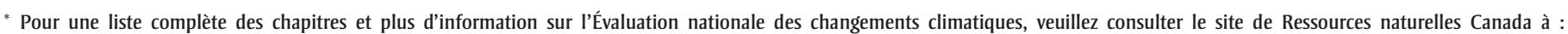
https://www.rncan.gc.ca/environnement/impacts-adaptation/19927

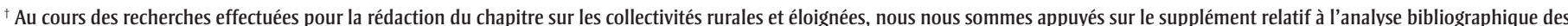

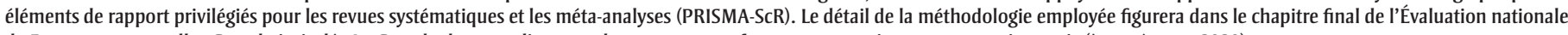
de Ressources naturelles Canada intitulée Le Canada dans un climat en changement : renforcer nos connaissances pour mieux agir (à paraître en 2020).
} 
de plus en plus extrêmes sont susceptibles d'entraîner une exacerbation des maladies chroniques et une incidence accrue des maladies infectieuses, en raison d'une plus grande exposition aux contaminants de l'environnement et aux maladies à transmission vectorielle ou d'origine hydrique ou alimentaire ${ }^{16}$, d'une contrainte accrue sur les affections chroniques sous-jacentes (p. ex. les maladies cardiovasculaires et respiratoires $)^{4,18}$ et d'une perturbation de la prestation des soins de santé et de la gestion des maladies chroniques ${ }^{6}$. Les recherches font également état d'un risque accru de maladies d'origine hydrique dans les régions rurales et éloignées à la suite d'événements de contamination liés aux phénomènes météorologiques ${ }^{5,25}$. En outre, les changements affectant les vents, les courants marins, les fleuves et les rivières risque d'acheminer des contaminants vers le Nord et ainsi d'augmenter la teneur en polluants organiques persistants et en métaux lourds toxiques des sources locales d'aliments et d'eau dans les régions polaires éloignées ${ }^{7,16,23}$, et on sait que la consommation de ces contaminants peut conduire à de nombreux problèmes de santé?

\section{Auǵmentation de l'incidence des blessures et des décès en lien avec les variations météorologiques}

Des conditions météorologiques extrêmes en rapide évolution, parmi lesquelles des vagues de chaleur, des tempêtes, des sécheresses, des inondations et la transformation des glaces maritimes, ont déjà des effets nocifs considérables sur la santé des populations des régions rurales et éloignées. Par exemple, la multiplication des épisodes de chaleur dans les régions rurales accroît le nombre de consultations en salle d'urgence dues aux coups de chaleur ou à des problèmes respiratoires ${ }^{26}$. De plus, les feux incontrôlés qui sévissent dans les collectivités forestières au Canada et les enjeux de santé qui en découlent (problèmes respiratoires, facteurs de stress pouvant nuire à la santé mentale et dégâts infligés aux infrastructures de santé essentielles) constituent des menaces à la sécurité et au bien-être des populations ${ }^{11,18,19}$.

\section{Impact des changements climatiques et des conditions environnementales imprévisibles sur la santé mentale et le bien-être}

$\mathrm{Au}$ fur et à mesure que l'environnement change et que la population doit s'adapter à de nouvelles conditions (le plus souvent moins enviables), on constate des perturbations de la santé mentale et du bien-être au sein des populations des régions rurales et éloignées. Par exemple, dans les communautés autochtones du Canada situées en région rurale ou éloignée, le bien-être des individus est généralement profondément lié au territoire, ce qui fait qu'une altération de l'environnement en lien avec les changements climatiques conduit à des perturbations dans l'accès aux lieux et aux pratiques culturellement importants ${ }^{3,6,12,27}$.

Pour les Inuits du Nunatsiavut en particulier, ces changements ont conduit à une augmentation de l'anxiété, de la peur, de la détresse, de la colère, du chagrin et de la dépression, du fait des modifications affectant les activités fondées sur le territoire, le lien à la terre et l'identité culturelle $e^{3,25,27}$. Dans les plans de développement régional au Manitoba, la perte potentielle des moyens de subsistance associée à la sécheresse est considérée comme un enjeu de santé mentale lié au climat ${ }^{28}$. Dans les provinces de l'Atlantique, l'augmentation

TABLEAU 1

Exemples de stratégies actuelles et de stratégies à mettre en place face aux effets nocifs des changements climatiques sur la santé dans les collectivités rurales et éloignées du Canada

\begin{tabular}{l} 
Exemples de stratégies d'adaptation déjà adoptées \\
\hline Mise en place de systèmes de production alimentaire locaux \\
Utilisation du savoir des collectivités locales issu de l'expérience au service de la \\
résilience des collectivités
\end{tabular}

Mise en place de projets de recherche et de programmes de veille au sein des collectivités afin de rassembler des données sur l'environnement et la santé en vue d'éclairer les processus décisionnels

Utilisation des connaissances autochtones et locales sur l'environnement physique, afin de prévenir les dangers et de se préparer adéquatement aux urgences

Utilisation d'une approche sociale du développement, engageant des professionnels de la santé, des travailleurs sociaux et des membres des professions axées sur la relation d'aide qui soutiennent les personnes subissant les effets directs des changements climatiques, afin de renforcer les moyens d'action des collectivités

Promotion des facteurs de protection de la santé physique et de la santé mentale par la perpétuation des liens avec l'enseignement axé sur le territoire et avec les arts et l'artisanat traditionnels, ainsi que par des occasions de rassemblement au sein des collectivités

Exemples de stratégies d'adaptation à mettre en place Références

$15,16,19$

$3,6,12$

Utilisation du savoir local, du savoir autochtone ou du savoir occidental afin de tenir compte des contextes socioculturels locaux particuliers

Élimination des obstacles sociaux à l'adaptation (p. ex. la pauvreté, les inégalités, les problèmes de logement, etc.) et réduction des facteurs non climatiques (p. ex. les maladies chroniques)

Utilisation de technologies novatrices (p. ex. la télésanté, les applications mobiles de surveillance, l'imagerie satellitaire)

$5-7,18,25$

Amélioration de la surveillance de la santé publique et étoffement des programmes de veille

Soutien aux pratiques de développement durable (p. ex. programmes d'énergie propre)

Amélioration des communications et sensibilisation accrue aux risques et aux mesures d'intervention pertinentes (p. ex. production de listes d'espaces sûrs et de livrets sur les foyers d'infection, élaboration d'une stratégie de diffusion)

Enrichissement des connaissances au sujet des impacts des changements climatiques sur la santé au moyen de recherches et d'investissements et partage des meilleures pratiques pertinentes pour l'adaptation du secteur de la santé publique aux changements

Renforcement des systèmes de santé et des mesures d'intervention d'urgence pour composer avec les risques climatiques et prendre les décisions appropriées (p. ex. création de guides techniques et de formations, intégration des données relatives aux changements climatiques dans les programmes d'études de médecine et de santé publique)

27

\section{Références}

$7,12,19,22$

5,22

5,19

$5,7,8,12,14,15,23,24,29$

$12,19,22,29$

$5,12,18,19,29$

$5,19,29$

14,19 
du nombre et de la gravité des tempêtes dans les collectivités côtières rurales a conduit selon certaines personnes à une dégradation d'importantes infrastructures en santé mentale ${ }^{29}$, ce qui varie généralement en fonction du sexe $\mathrm{s}^{30}$.

\section{Mesures d'adaptation aux changements climatiques et bénéfices pour les régions rurales et éloignées}

Au-delà des nombreux défis que nous venons de mentionner, se centrer sur l'adaptation aux changements climatiques et prendre la mesure des bénéfices liés à l'atténuation de ces changements offre des opportunités au secteur de la santé. De nombreuses collectivités canadiennes rurales et éloignées ont d'ailleurs déjà commencé à élaborer et à mettre en œuvre des stratégies d'adaptation en matière de santé (tableau 1). Une réponse optimale aux effets des changements climatiques sur la santé exige de modifier les stratégies actuelles et d'adopter un certain nombre de nouvelles mesures, notamment l'emploi de multiples systèmes de connaissances relevant des divers contextes socioculturels, l'analyse des facteurs non climatiques ayant un effet sur l'adaptation, l'utilisation de technologies novatrices, l'amélioration de la veille sanitaire et son intégration aux activités de surveillance environnementale, la promotion de pratiques de développement durable, une meilleure sensibilisation aux risques et aux mesures d'intervention pertinentes, une diffusion des connaissances sur les impacts des changements climatiques et enfin le renforcement des moyens d'action du secteur de la santé face aux changements climatiques (tableau 1). Pour que les collectivités rurales et éloignées puissent continuer à s'adapter aux impacts des changements climatiques sur la santé, il importe également de tenir compte de leurs composantes socioculturelles, économiques et géographiques locales et régionales, de favoriser et d'utiliser l'expertise des individus et des collectivités de ces régions ainsi que de continuer à replacer la santé des populations au sein du contexte socioculturel et matériel que constituent ces régions (figure 1).

\section{Remerciements}

Nous tenons à remercier Don Lemmen, Fiona Warren et l'équipe de direction de l'évaluation nationale de Ressources naturelles Canada, Le Canada dans un climat en changement : renforcer nos connaissances pour mieux agir. Nous souhaitons aussi remercier les collectivités rurales et éloignées ainsi que les chercheurs et les décideurs dont les connaissances et l'expertise ont contribué à éclairer la rédaction de cet aperçu.

\section{Conflits d'intérêts}

Les auteurs déclarent n’avoir aucun conflit d'intérêts.

FIGURE 1

Principaux enjeux en matière de santé et principales stratégies d'adaptation dans le contexte des changements climatiques au sein des régions rurales et éloignées du Canada

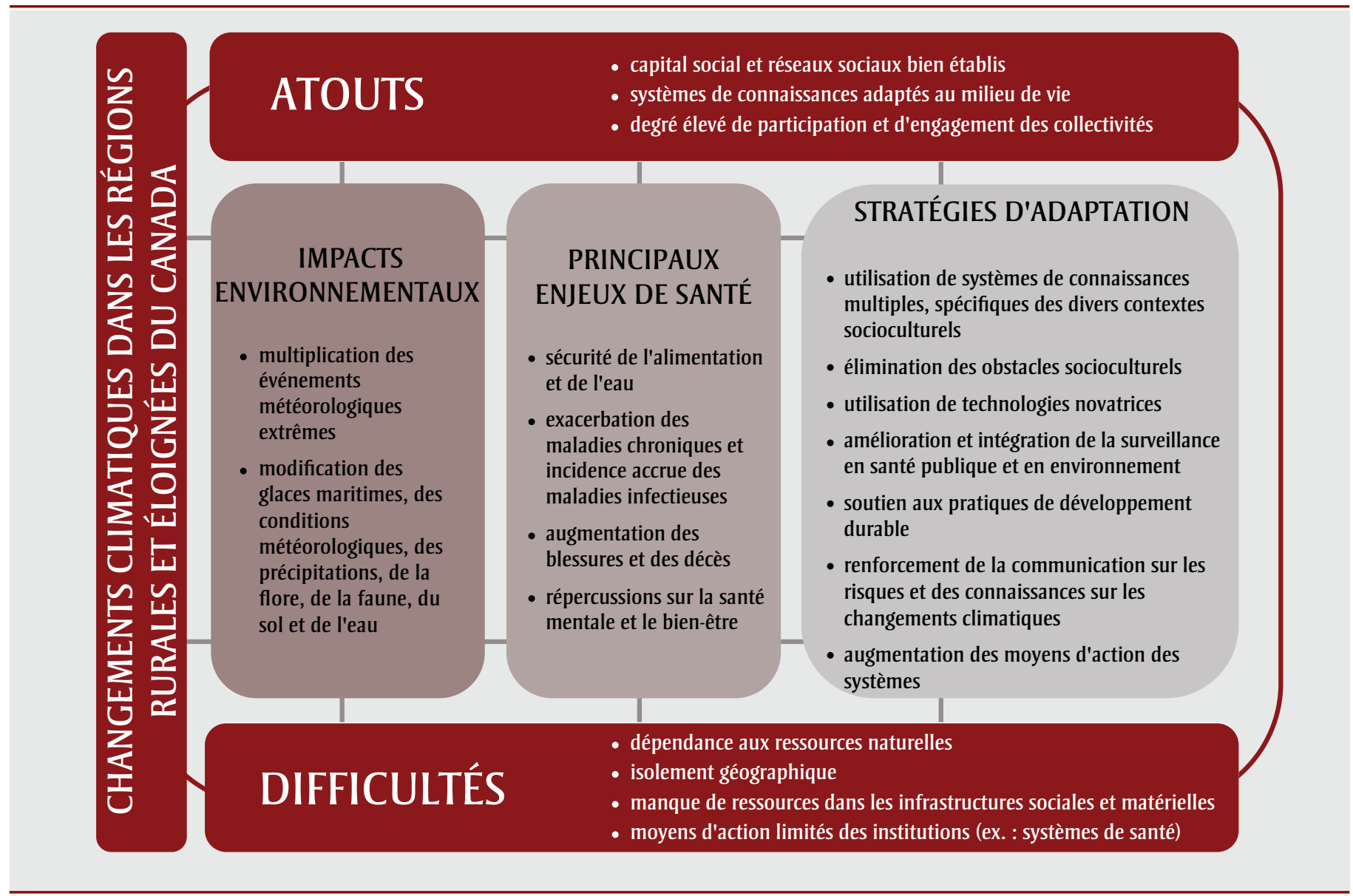




\section{Contributions des auteurs et avis}

AC, SLH et KV ont contribué à la conception de l'analyse documentaire et de la chaîne de recherche visant à obtenir des documents parallèles et des documents évalués par des pairs. NK et SM ont effectué la recherche des documents pertinents et extrait les données quantitatives des articles trouvés. AK, AC et SLH ont fait une analyse qualitative des articles et résumé l'information; ils ont entre autres relevé les thèmes émergents sur les enjeux de santé et les stratégies d'adaptation et ont également rédigé l'article. Tous les auteurs ont participé à la révision du manuscrit.

Le contenu de l'article et les points de vue qui y sont exprimés n'engagent que les auteurs; ils ne correspondent pas nécessairement aux points de vue du gouvernement du Canada.

\section{Références}

1. Intergovernmental Panel on Climate Change. Climate Change 2014: Impacts, Adaptation, and Vulnerability, Part B: Regional Aspects. Contribution of Working Group II to the Fifth Assessment Report of the Intergovernmental Panel on Climate Change (sous la direction de Barros VR, Field CB, Dokke JD, et al.). Cambridge (UK), New York (USA) : Cambridge University Press; 2014. 688 p.

2. Intergovernmental Panel on Climate Change. Climate Change 2014: Impacts, Adaptation, and Vulnerability, Part A: Global and Sectoral Aspects. Contribution of Working Group II to the Fifth Assessment Report of the Intergovernmental Panel on Climate Change (sous la direction de Field CB, Barros VR, Dokken DJ, et al.). Cambridge (UK), New York (USA): Cambridge University Press; 2014. 1132 p.

3. Cunsolo A, Ellis NR. Ecological grief as a mental health response to climate change-related loss. Nat Clim Chang. 2018;8(4):275-281. doi: 10.1038/s41558 $-018-0092-2$.

4. Agence de la santé publique du Canada. Les incidences des changements climatiques sur la santé de la population canadienne [Internet]. Ottawa (Ont.) : Agence de la santé publique du Canada; 2017. En ligne à : http://publications.gc.ca/collections /collection_2017/aspc-phac/HP5-122 -2017-fra.pdf
5. Groupe d'experts sur les résultats de l'adaptation et de la résilience. Mesure des progrès en matière d'adaptation et de résilience climatique : recommandations à l'intention du gouvernement [Internet]. Gatineau (Québec) : Environnement et Changement climatique Canada; 2017. En ligne à :http:// publications.gc.ca/collections/collection _2018/eccc/En4-329-2018-fra.pdf

6. Cunsolo Willox A, Stephenson E, Allen J, et al. Examining relationships between climate change and mental health in the Circumpolar North. Reg Environ Chang. 2015;15(1):169-182.

7. Berner J, Brubaker M, Revitch B, Kreummel E, Tcheripanoff M, Bell J. Adaptation in Arctic circumpolar communities: food and water security in a changing climate. 2016;1:1-8.

8. Durkalec A, Furgal C, Skinner MW, Sheldon T. Climate change influences on environment as a determinant of Indigenous health: relationships to place, sea ice, and health in an Inuit community. Soc Sci Med. 2015;136137:17-26.

9. Ressources naturelles Canada. Le Canada dans un climat en changement [Internet]. Ottawa (Ont.) : Ressources naturelles Canada; 2018 [consulté le 26 nov. 2018]. En ligne à : https:// www.rncan.gc.ca/environnement /impacts-adaptation/19923

10. Rapaport E, Manuel P, Krawchenko T, Keefe J. How can aging communities adapt to coastal climate change? Planning for both social and place vulnerability. Can Public Policy. 2015; 41(2):166-177.

11. Government of Saskatchewan. Prairie resilience: a made-in-Saskatchewan climate change strategy [Internet]. Government of Saskatchewan; 2017. En ligne à :http://publications.gov .sk.ca/documents/66/104890-2017 Climate Change Strategy.pdf

12. Ford JD, Willox AC, Chatwood S, et al. Adapting to the effects of climate change on Inuit health. Am J Public Health. 2014;104(Suppl. 3):e9-17.
13. Clark DG, Ford JD. Emergency response in a rapidly changing Arctic. Can Med Assoc J. 2017;189:e135-136.

14. Young SK, Tabish TB, Pollock NJ, Kue Young T. Backcountry travel emergencies in arctic Canada: a pilot study in public health surveillance. Int $\mathrm{J}$ Environ Res Public Health. 2016;13(3): 276.

15. Barbeau CD, Oelbermann M, Karagatzides JD, Tsuji LJS. Sustainable agriculture and climate change: producing potatoes (Solanum tuberosum L.) and bush beans (phaseolus vulgaris L.) for improved food security and resilience in a Canadian subarctic first nations community. Sustain. 2015;7(5):5664-5681.

16. Loring PA, Gerlach SC. Searching for progress on food security in the North American North: a research synthesis and meta-analysis of the peerreviewed literature. Arctic. 2015;68(3): 380-392.

17. Harper SL, Edge VL, Schuster-Wallace CJ, Berke O, McEwen SA. Weather, water quality and infectious gastrointestinal illness in two Inuit communities in Nunatsiavut, Canada: potential implications for climate change. Ecohealth. 2011;8(1):93-108.

18. Dodd W, Scott P, Howard C, et al. Lived experience of a record wildfire season in the Northwest Territories, Canada. Can J Public Heal. 2018; 109(3):327-337.

19. Gouvernement des Territoires du Nord-Ouest. Cadre stratégique sur le changement climatique des TNO 2030 [Internet]. Gouvernement des Territoires du Nord-Ouest; 2017. En ligne à :https://www.enr.gov.nt.ca /sites/enr/files/resources/128-climate _change_strategic_framework_web .pdf

20. Statham S, Ford J, Berrang-Ford L, Lardeau MP, Gough W, Siewierski R. Anomalous climatic conditions during winter 2010-2011 and vulnerability of the traditional Inuit food system in Iqaluit, Nunavut. Polar Rec (Gr Brit). 2015;51(3):301-317. 
21. Krawchenko T, Keefe J, Manuel P, Rapaport E. Coastal climate change, vulnerability and age friendly communities: linking planning for climate change to the age-friendly communities' agenda. J Rural Stud. 2016;44: 55-62. doi: 10.1016/j.jrurstud.2015 .12 .013 .

22. Drolet JL, Sampson T. Addressing climate change from a social development approach: small cities and rural communities' adaptation and response to climate change in British Columbia, Canada. Int Soc Work. 2017;60(1): 61-73.

23. Medeiros AS, Wood P, Wesche SD, Bakaic M, Peters JF. Water security for northern peoples: review of threats to Arctic freshwater systems in Nunavut, Canada. Reg Environ Chang. 2017; 17(3):635-647.

24. Bakaic M, Medeiros AS. Vulnerability of northern water supply lakes to changing climate and demand [Internet]. Arct Sci. 2017;3(1):1-16. En ligne à : http://www.nrcresearchpress.com /doi/10.1139/as-2016-0029

25. Harper SL, Edge VL, Ford J, et al. Climate-sensitive health priorities in Nunatsiavut, Canada. BMC Public Health. 2015;15(1):605.

26. Bishop-Williams KE, Berke O, Pearl DL, Kelton DF. A spatial analysis of heat stress related emergency room visits in rural Southern Ontario during heat waves. BMC Emerg Med. 2015; 15(1):1-9. doi: 10.1186/s12873-015 $-0043-4$.

27. Cunsolo A, Shiwak I, Wood M. "You Need to Be a Well-Rounded Cultural Person": youth mentorship programs for cultural preservation, promotion, and sustainability in the Nunatsiavut Region of Labrador. Dans : Fondahl G, Wilson G (dir.), Northern Sustainabilities: Understanding and Addressing Change in the Circumpolar World. Springer Polar Sciences. 2017:285-303.

28. Government of Manitoba. A made-inManitoba climate and green plan. Hearing from Manitobans [Internet]. Government of Manitoba; 2017. En ligne à : http://www.gov.mb.ca/asset _library/en/climatechange/climate greenplandiscussionpaper.pdf
29. Province du Nouveau-Brunswick. La transition vers une économie à faibles émissions de carbone : Le plan d'action du Nouveau-Brunswick sur les changements climatiques. Fredericton (N.-B.), Province du NouveauBrunswick, 2016. En ligne à : https:// www2.gnb.ca/content/dam/gnb /Departments/env/pdf/ClimateClimatiques/LaTransitionVersUne EconomieAFaiblesEmissionsDe Carbone.pdf

30. Vasseur L, Thornbush M, Plante S. Gender-based experiences and perceptions after the 2010 winter storms in Atlantic Canada. Int $\mathrm{J}$ Environ Res Public Health. 2015;12(10):12518-12529.

31. Agence de la santé publique du Canada. Le groupe de réflexion rural 2005 comprendre les problèmes qui se posent aux familles des communautés rurales et éloignées [Internet]. Ottawa (Ont.) : Agence de la santé publique du Canada; 2007. En ligne à : http:// www.phac-aspc.gc.ca/hp-ps/dca-dea /publications/rtt-grr-2005/2-fra.php 